\title{
Article \\ SIR-solution for slowly time-dependent ratio between recovery and infection rates
}

\author{
Martin Kröger ${ }^{1 *(D)}$ and Reinhard Schlickeiser $2,3 *$ (D) \\ 1 Polymer Physics, Department of Materials, ETH Zurich, Zurich CH-8093, Switzerland; mk@mat.ethz.ch \\ 2 Theoretische Physik, Lehrstuhl IV: Weltraum- und Astrophysik, Ruhr-Universität Bochum, D-44780 Bochum, \\ Germany; rsch@tp4.rub.de \\ 3 Institut für Theoretische Physik und Astrophysik, Christian-Albrechts-Universität zu Kiel, Leibnizstr. 15, \\ D-24118 Kiel, Germany \\ * Correspondence: mk@mat.ethz.ch (M.K.),rsch@tp4.rub.de (R.S.)
}

\begin{abstract}
The temporal evolution of pandemics described by the susceptible-infectious-recovered (SIR)-compartment model is sensitively determined by the time dependence of the infection $(a(t))$ and recovery $(\mu(t))$ rates regulating the transitions from the susceptible to the infected and from the infected to the recovered compartment, respectively. Here approximated SIR-solutions for different time dependencies of the infection and recovery rates are derived which are based on the adiabatic approximation assuming time-dependent ratios $k(t)=\mu(t) / a(t)$ varying slowly in comparison to the typical time characteristics of the pandemic wave. For such slow variations the available analytical approximations from the KSSIR-model, valid for a stationary value of the ratio $k$, are used to insert a-posteriori the adopted time-dependent ratio of the two rates. Instead of investigating endless different combinations of the time dependencies of the two rates $a(t)$ and $\mu(t)$ a suitably parameterized reduced time dependence of the ratio $k(\tau)$ is adopted. Together with the definition of the reduced time this parameterized ratio $k(\tau)$ allows us to cover a great variety of different time dependencies of the infection and recovery rates. The agreement between the solutions from the adiabatic approximation in its four different studied variants and the exact numerical solutions of the SIR-equations is remarkably good providing strong confidence in the accuracy of the proposed adiabatic approximation.
\end{abstract}

Keywords: epidemiology; statistical analysis; time-scale separation; differential equations; adiabatic approximation

\section{Introduction}

About a century ago the susceptible-infectious-recovered/removed (SIR) model has been introduced [1] to the mathematical theory of epidemics. Together with later improvements [2] it represents the fundamental compartment model where any considered population of $N$ persons is divided into the three fractions of susceptible (S), infected (I) and recovered/removed $(R)$ persons. The infection rate $a(t)$ and the recovery/removed rate $\mu(t)$ regulate the transitions from the susceptible to the infected compartment and from the infected to the recovered/removed compartment, respectively, see Eqs. (1) below. The original SIR model has been generalized to more complicated version adding additional compartments such as the fraction of exposed persons (for reviews see [3,4]) and vaccinated persons [5], and widely applied to epidemic outbreaks including Covid-19 and SARS-CoV-2 [6].

Very often the SIR equations (1) are solved numerically with adopted stationary infection $\left(a_{0}\right)$ and recovery $\left(\mu_{0}\right)$ rates so that their ratio $k=\mu_{0} / a_{0}$ is also constant and stationary, although an analytical solution in terms of an inverse integral of this case is known since 2014 [7].

Analytical solutions for arbitrary but given time dependencies of the infection rate $a(t)$ were derived recently $[8,9]$ for the infinite time-domain and the semi-time time domain for the case of a stationary ratio $k=\mu(t) / a(t)$, implying that the recovery rate has exactly the same time dependence as the infection rate. Hereafter we refer to these generalizations 
as the KSSIR-model. This generalization to a time-dependent infection rate is important as such time-dependencies are caused by non-pharmaceutical interventions (NPIs) taken during pandemic outbreaks. In the KSSIR-model [8,9] exact analytical inverse solutions $t(Q)$ for all relevant quantities $Q \in[S, I, R]$ in terms of Lambert functions were obtained. These inverse solutions were approximated with high accuracy yielding the explicit timedependencies $Q(t)$ by inverting the Lambert functions. Of particular importance are the obtained analytical expressions for the peak time and asymptotic behaviors, early doubling times, late half decay time of the rate of new infections $\dot{J}(t)=a(t) S(t) I(t)$ and its corresponding cumulative number $J(t)$, which allowed the quantitative comparison with the monitored temporal evolution of different waves the Covid-19 [10] and the forecast for the omicron mutant [11].

It is the purpose of the present manuscript to investigate analytical solutions of the SIR equations for different time dependencies of the infection $(a(t))$ and recovery $(\mu(t)$ rates so that their ratio no longer is constant and becomes also time-dependent. To the best of our knowledge this general case has not been studied in the literature apart from [12] who has studied special analytical solutions based on non-constant ratios $k(S)$ being of polynomial form of order $S^{4}$ or less in the case of positive or negative powers. However, in this work no relations to monitored pandemic parameters have been established.

Here we follow a different approach by investigating the adiabatic case which refers to slowly varying (in comparison to the typical time characteristics of the pandemic wave) time-dependent ratios $k(t)$. The adiabatic approximation $[13,14]$ is an established wellknown method in theoretical physics especially semi-classical quantum mechanics see e.g. the WKBJ-method [15-18] for slowly varying potentials or the Born-Oppenheimerapproximation [19] for the analysis of molecules. The latter simplifies the solution of the quantum mechanical equations of motion by taking advantage of the drastically different time scales of motion of the light and heavy particles in the system. Thus for the analysis below for slow variations of the function $k(t)$ we will use the available analytical approximations from the KSSIR-model (which strictly are valid only for constant ratios $k$ ) and insert a-posteriori the adopted time-dependent ratio $k(t)$. The resulting adiabatic solutions will then be compared to the exact numerical solutions of the SIR-equations (4) for given time variations of the ratio $k(t)$.

\section{Starting equations}

The general dynamical SIR equations for the fractions of susceptible (S), infectious (I), and recovered/removed $(R)$ compartments are

$$
\begin{aligned}
\dot{S}(t) & =-a(t) S(t) I(t), \\
\dot{I}(t) & =a(t) S(t) I(t)-\mu(t) I(t), \\
\dot{R}(t) & =\mu(t) I(t),
\end{aligned}
$$

which obey the sum constraint

$$
S(t)+I(t)+R(t)=1 .
$$

We consider the semi-time case with the initial conditions $S\left(t=t_{0}\right)=1-\eta, I\left(t=t_{0}\right)=\eta$ and $R\left(t=t_{0}\right)=0$. It is convenient to introduce the reduced time variable

$$
\tau=\int_{t_{0}}^{t} d y a(y),
$$

which can be calculated for any given time-dependence of the infection rate $a(t)$. Equations (1) then read

$$
\frac{d I}{d \tau}=S I-k(\tau) I, \quad \frac{d S}{d \tau}=-S I, \quad \frac{d R}{d \tau}=k(\tau) I,
$$




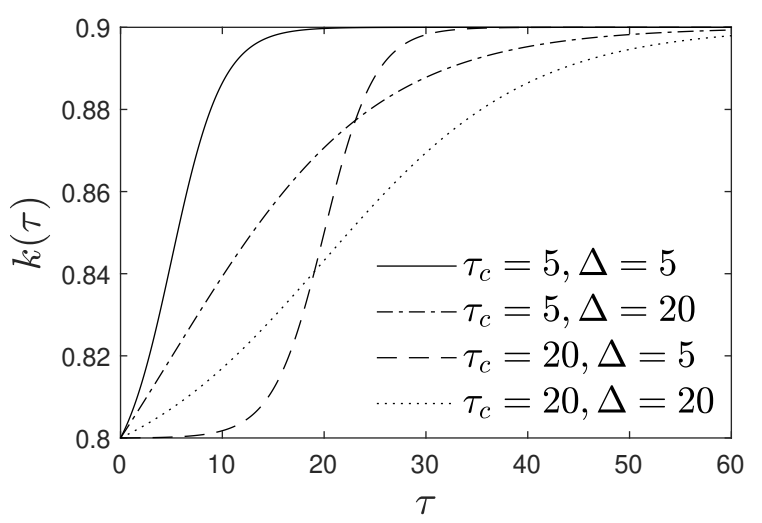

Figure 1. The function $k(\tau)$ stated in Eq. (6) as a function of $\tau$ for the initial and final values $k_{0}=0.8$ and $k_{1}=0.9$ for different choices of its parameters $\tau_{c}$ and $\Delta$.

in terms of the dimensionless, time-dependent ratio of infection to recovery rate

$$
k(\tau(t))=\frac{\mu(t(\tau))}{a(t(\tau))}
$$

There are no obvious natural choices for the real time dependencies of the infection $(a(t)$ and the recovery $(\mu(t))$ rates, and they may differ from mutant to mutant. Initially at the start of the mutant outbreak without any taken non-pharmaceutical interventions (NPIs) both rates have the values $a\left(t_{0}\right)=a_{0}$ and $\mu\left(t_{0}\right)=\mu_{0}$. Dedicated medication of infected persons certainly will increase the recovery rate from its initial value, whereas the NPIs [20-28] (such as social distancing, quarantining and mask obligations) effectively reduce the infection rate from its initial value.

Instead of investigating endless different combinations of the time dependencies of the two rates $a(t)$ and $\mu(t)$ we follow a different approach by adopting a suitably parameterized reduced time dependence of the ratio $k(\tau)$. Together with Eq. (3), holding for any given real time dependence of the infection rate $a(t)$, we are then able to represent quite a variety of different real time dependencies of the infection $(a(t))$ and recovery rate $(\mu(t))$ as we detail in Sect. $V$ below. As suitably parameterized reduced time dependency we adopt

$$
k(\tau)=k_{1}+\frac{k_{1}-k_{0}}{1+\tanh \left(\frac{\tau_{c}}{\Delta}\right)}\left[\tanh \left(\frac{\tau-\tau_{c}}{\Delta}\right)-1\right] .
$$

The function (6) starts from the value $k_{0}$ at the initial time $\tau=0$ and approaches the final value $k_{1}$ after infinite time $\tau=\infty$. As Fig. 1 demonstrates its parameters $\tau_{c}$ and $\Delta$ regulate the sharpness of the transition from $k_{0}$ to $k_{1}$. For small differences between the chosen initial $\left(k_{0}\right)$ and final $\left(k_{1}\right)$ values of the function $k(\tau)$ the overall variation of this function is comparatively small. The last argument can be formulated more quantitatively. The function (6) has its strongest gradient

$$
\left(\frac{d k}{d \tau}\right)_{\max }=\frac{k_{1}-k_{0}}{\left(1+\tanh \frac{\tau_{c}}{\Delta}\right) \Delta}
$$

at $\tau=\tau_{c}$ so that its shortest relative variation time scale is given by

$$
\tau_{k, \min }=\left|\frac{k\left(\tau_{c}\right)}{\left(\frac{d k}{d \tau}\right)_{\max }}\right|=\frac{\left[k_{0}+k_{1} \tanh \left(\frac{\tau_{c}}{\Delta}\right)\right] \Delta}{\left|k_{1}-k_{0}\right|}>\frac{k_{0} \Delta}{\left|k_{1}-k_{0}\right|},
$$

where the last lower limit holds because $\tanh \left(\tau_{c} / \Delta\right)>0$ for all finite values of the parameters $\Delta$ and $\tau_{c}$. Quantitatively, a slowly varying function $k(\tau)$ means that the lower limit (8) 
of relative time scale is long compared to the typical relative time variation of the pandemic wave which we characterized by its early doubling time [29] $\tau_{2}=(\ln 2) /\left(1-k_{0}\right)$, implying $k_{0} \Delta /\left|k_{1}-k_{0}\right| \gg \tau_{2}$, or

$$
\frac{k_{0}\left(1-k_{0}\right) \Delta}{\left|k_{1}-k_{0}\right|} \gg 0.693
$$

as quantitative parameter requirement for the slowliness of the time variation of the function $k(\tau)$. The time scale (8) increases inversely to the difference between the chosen initial $\left(k_{0}\right)$ and final $\left(k_{1}\right)$ values of the function $k(\tau)$. For all examples considered below the parameter requirement (9) is well satisfied.

\section{Analytical adiabatic approximations}

We use the exact and analytical results from the KSSIR model summarized in Sect. 2 of ref. [11] and insert a-posteriori the reduced time-dependence (6). Consequently, we obtain for this adiabatic approximation that $S(\tau)=1-J(\tau), I(\tau)=J(\tau)+k(\tau) \epsilon+k(\tau) \ln [1-$ $J(\tau)], R(\tau)=-k(\tau)[\epsilon+\ln (1-J(\tau))]$ with $\epsilon=-\ln (1-\eta)$ in terms of the cumulative number of new infections $J(\tau)$. The latter is given by [10]

$$
J(\tau) \simeq \begin{cases}\eta+\frac{J_{0}(k)-\eta}{1+\sqrt{\frac{\max (k)}{c_{0}} \frac{\sinh \left[c_{3}\left(\hat{t}_{m}-\tau\right)\right]}{\sinh \left(c_{3} \tau\right)}}} & \text { for } \tau \leq \hat{\tau}_{m}, \\ J_{\infty}\left(k_{1}\right)-\frac{J_{\infty}\left(k_{1}\right)-J_{0}(k)}{\frac{J_{m}(k)}{c_{4}\left[I_{\infty}\left(k_{1}\right)-J_{0}(k)\right]}\left[e^{c_{4}\left(\tau-\hat{\tau}_{m}\right)}-1\right]+1} & \text { for } \tau \geq \hat{\tau}_{m}\end{cases}
$$

where $k, c_{3}$ and $c_{4}$ stand for $k(\tau), c_{3}(k(\tau))$ and $c_{4}(k(\tau))$. The interpolated dimensionless peak time is given by

$$
\begin{aligned}
\hat{\tau}_{m} & =\frac{2}{\tau_{m}^{-1}\left(k_{0}\right)+\tau_{m}^{-1}\left(k_{1}\right)} \\
\tau_{m}(k) & =\frac{1}{c_{3}(k)} \operatorname{artanh}\left[\frac{2 c_{3}(k)}{c_{1}(k)+\frac{2 c_{0}}{J_{0}(k)-\eta}}\right],
\end{aligned}
$$

and the abbreviations

$$
\begin{aligned}
c_{0} & =\eta(1-\eta), \\
c_{1}(k) & =1-k-2 \eta, \\
c_{2}(k) & =\frac{j_{\max }(k)-c_{0}-c_{1}(k)\left[J_{0}(k)-\eta\right]}{\left[J_{0}(k)-\eta\right]^{2}} \\
c_{3}(k) & =\sqrt{\left(\frac{c_{1}(k)}{2}\right)^{2}-c_{0} c_{2}(k),} \\
c_{4}(k) & =J_{\infty}\left(k_{1}\right)-(1-k) .
\end{aligned}
$$

Here

$$
j_{\max }(k)=\frac{k^{2}}{4}\left\{\left[1+W_{-1}\left(-\frac{2(1-\eta)}{k e^{1+\frac{1}{k}}}\right)\right]^{2}-1\right\}
$$

denotes the maximum rate of new infections occurring at

$$
J_{0}(k)=1+\frac{k}{2} W_{-1}\left(-\frac{2(1-\eta)}{k e^{1+\frac{1}{k}}}\right)
$$

and

$$
J_{\infty}\left(k_{1}\right)=\lim _{\tau \rightarrow \infty} J(\tau) \simeq 1+k_{1} W_{0}\left(-\frac{1-\eta}{k_{1} e^{\frac{1}{k_{1}}}}\right)
$$


(I)
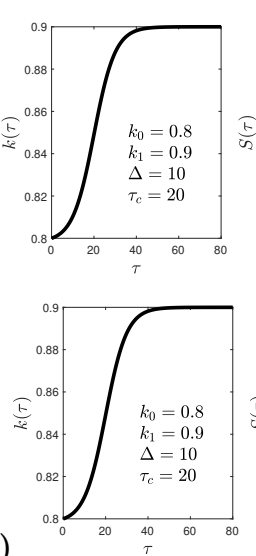

(II)

(III)
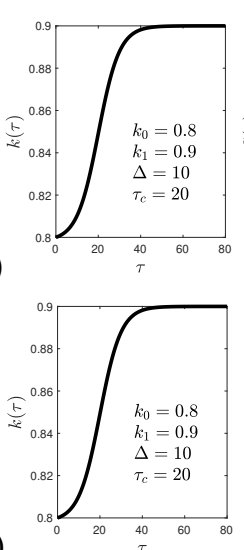
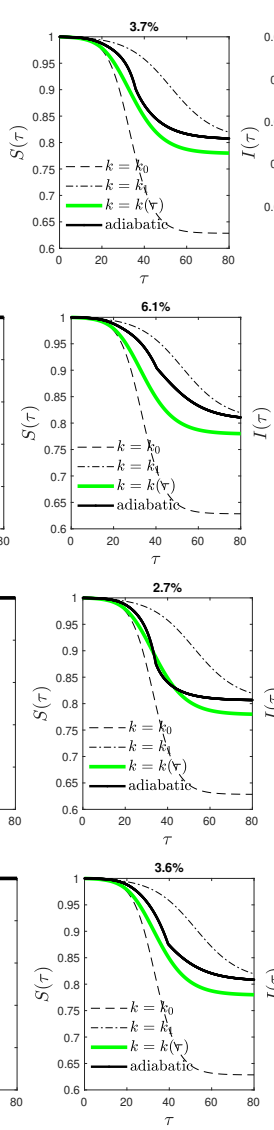

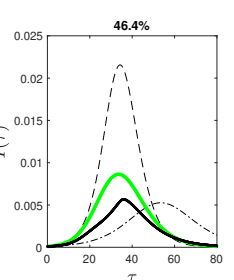

$52.4 \%$

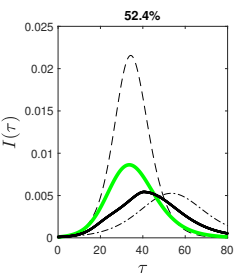

$43.9 \%$

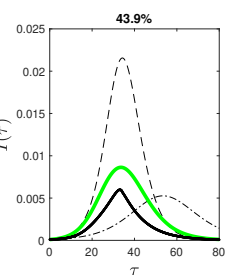

43.4\%

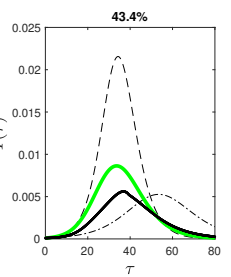

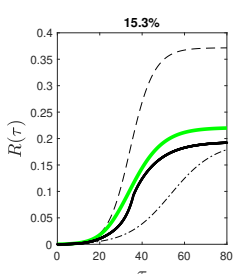

$27.8 \%$

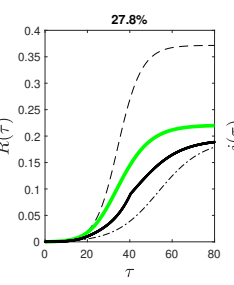

$12.2 \%$

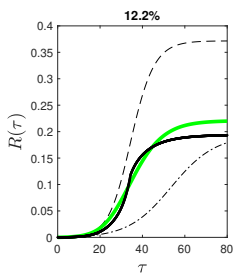

$15.8 \%$

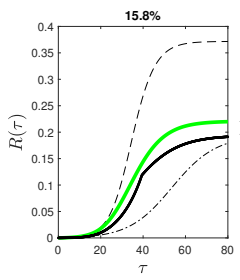

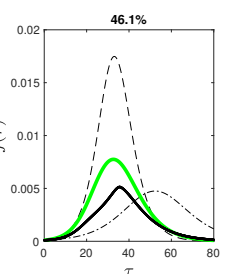

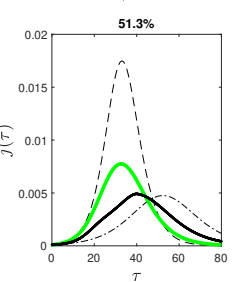

42.3

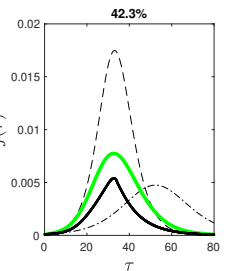

$42.9 \%$

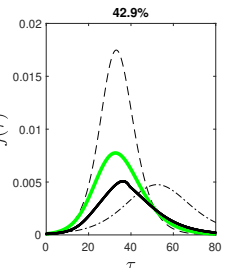

Figure 2. Results for all four versions I-IV. Parameters mentioned in figure, $\eta=10^{-4}$.

is the final cumulative fraction of infected persons. Equations (13)-(15) depend on the principal $\left(W_{0}\right)$ and non-principal $\left(W_{-1}\right)$ solution of Lambert's equation [8], the well-known and documented Lambert functions. We emphasize that for small values of $\eta \ll 1$ the results (13)-(15) are basically independent of the value of $\eta$ and only determined by the ratio $k(\tau)$.

The reduced time dependence of the rate of new infections in the adiabatic case is then given by

$$
j(\tau) \simeq[1-J(\tau)][J(\tau)+k(\tau) \epsilon+k(\tau) \ln (1-J)] .
$$

We refer to Eqs. (11)-(16) as version I of the adiabatic analytical approximation. Additionally we investigate three slightly different versions of this model: versions II to IV. In Version II we calculate the $j_{\max }$ not with its own equation (13), but using Eq. (16) with $J$ replaced by $J_{0}$ from Eq. (14). For version III we use

$$
\hat{J}_{0}=\frac{2}{\left[J_{0}^{-1}\left(k_{0}\right)+J_{0}^{-1}\left(k_{1}\right)\right]}
$$

instead of $J_{0}(k)$ in (14) within the spirit of the interpolated $\hat{\tau}_{m}$ in Eq. (11). This appears more consistent, as the $\hat{\tau}_{m}$ belongs to the value of $J_{0}$. Version IV combines versions II and III.

\section{Comparison of analytical and exact results in reduced time}

In Figs. 2-8 we compare the exact numerical solutions with the four versions of the analytical adiabatic approximations for different parameter choices of the reduced time dependence of the ratio (6). The numerical solution is obtained using a solver for moderately stiff ordinary differential equations, as proposed earlier [30]. To this end we use an implementation of the trapezoidal rule using a "free" interpolant [31,32]. 
(I)

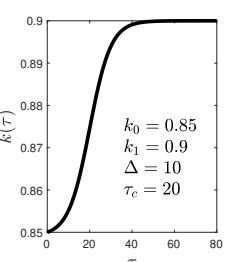

(II)
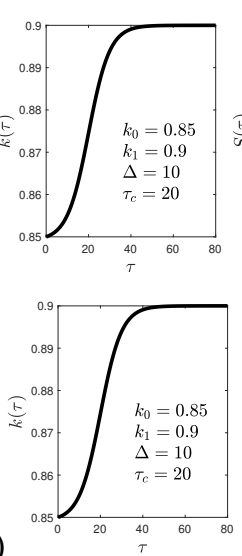

(III)

(IV)

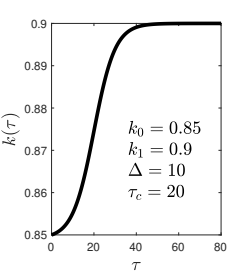

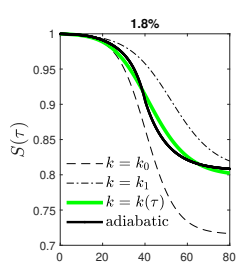
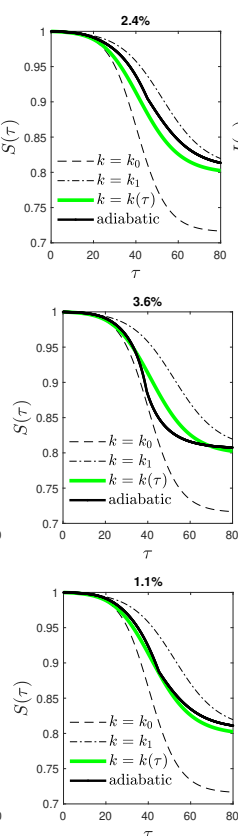
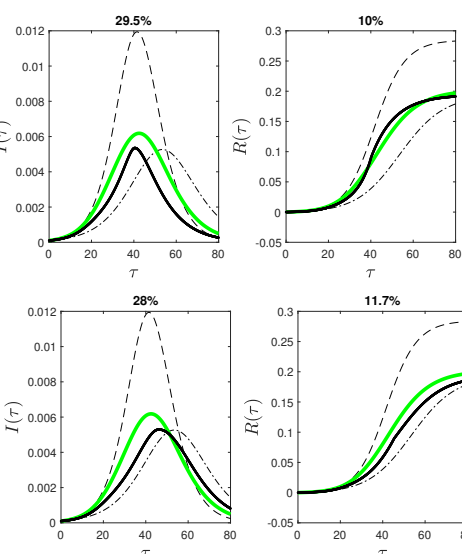

43.6\%
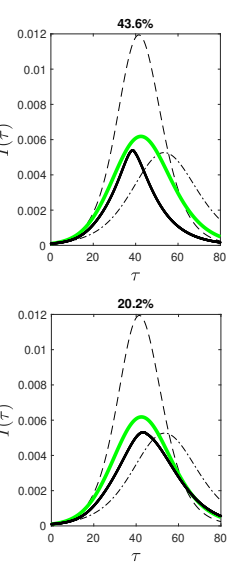

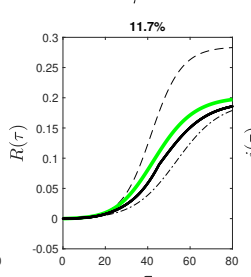

$19.3 \%$
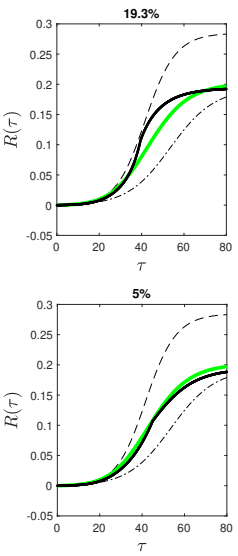
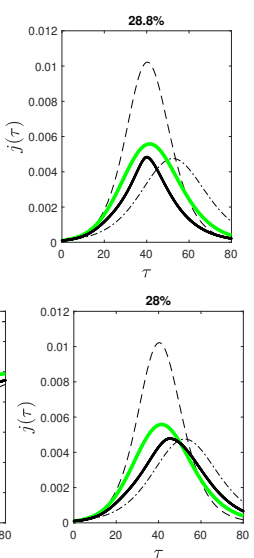

42.9\%

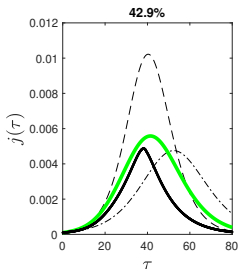

$20.4 \%$

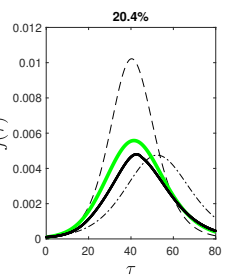

Figure 3. Results for all four versions I-IV. Parameters mentioned in figure.

In each left panel the chosen parameters of the ratio (6) and its reduced time dependence is shown. Only in Fig. 7a decreasing ratio with reduced time is chosen whereas in Figs. 2-4 and 5-8 increasing ratios are considered. In each figure the second, third, fourth and fifth from left panels display the calculated dependence of the fractions $S(\tau), I(\tau)$, $R(\tau)$ and the rate of new infections $j(\tau)$ as a function of the reduced time $\tau$. In every panel the green curve provides the exact numerical result, the black curve shows the adiabatic approximation, the dashed curve shows the KSSIR-variation adopting the initial ratio $k_{0}$ at all times, and the dot-dashed curve displays the KSSIR-variation adopting the final ratio $k_{1}$ at all times. As expected in every case both the exact numerical curve and the adiabatic approximation initially at small reduced times are close to the KSSIR-behavior for the value $k_{0}$, whereas at late times these two curves approximate the KSSIR-behavior for the final value $k_{1}$.

The agreement between the exact numerical reduced time and the adiabatic approximation reduced time dependence in all four versions is remarkably good. The maximum relative deviations between exact (green) and approximate (black) solutions are stated in the title of each figure. In the worst case the maximum deviation is $68.1 \%$ but in most shown examples much less. This remarkable good agreement provides strong confidence in the accuracy of the proposed adiabatic approximation.

Apparently, there are no strong differences in the four versions of the adiabatic approximation. However, putting the highest emphasis on the rates of new infections $j(\tau)$, as these can be compared with the monitored pandemic data in different countries and societies, version IV of the adiabatic approximation gives the most accurate analytical results, and therefore may be favored over the other three versions. Regarding the rate $j(\tau)$ the maximum deviation increases roughly as $\simeq 400\left|k_{1}-k_{0}\right|$ percent with greater differences in the final and initial values of the function $k(\tau)$. 
(I)

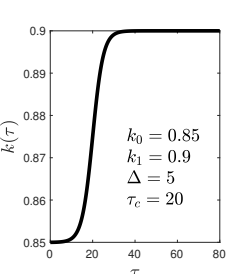

(II)

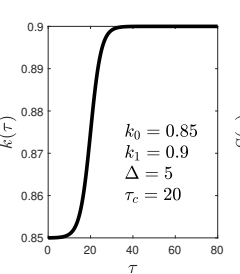

(III)
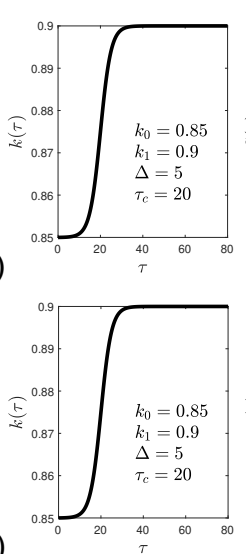
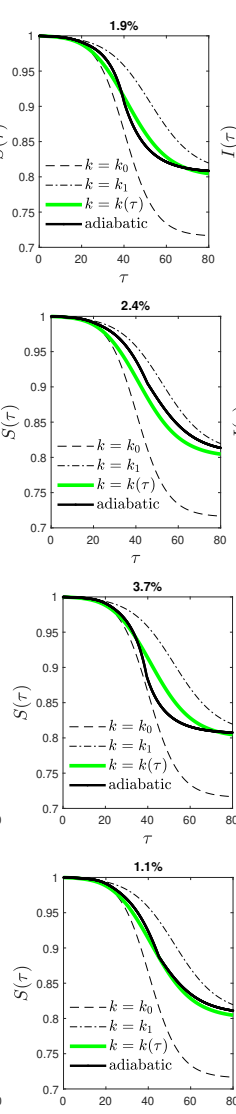

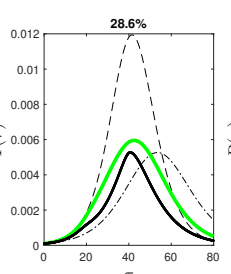

29.3\%

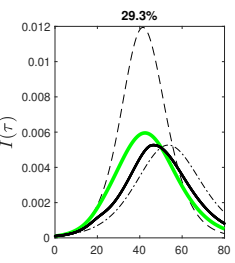

$43 \%$
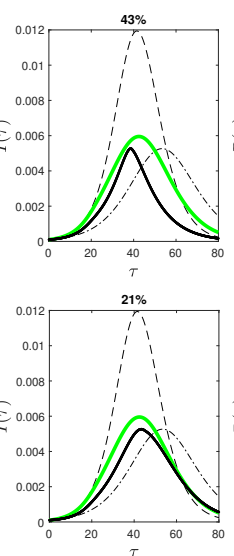

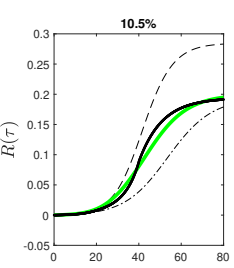

$11.8 \%$
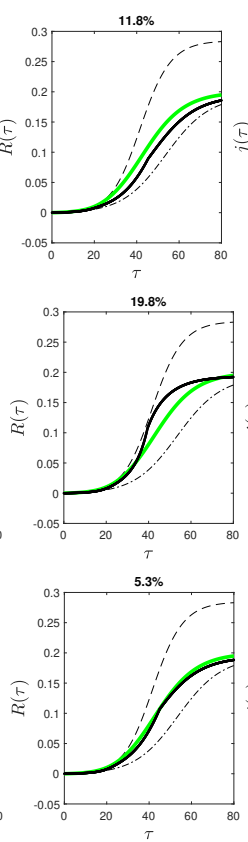

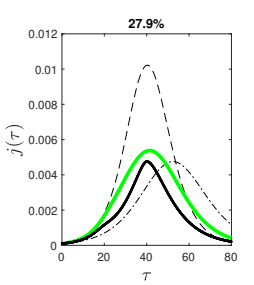

29.7

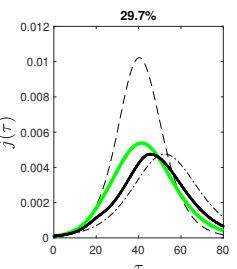

$42.4 \%$
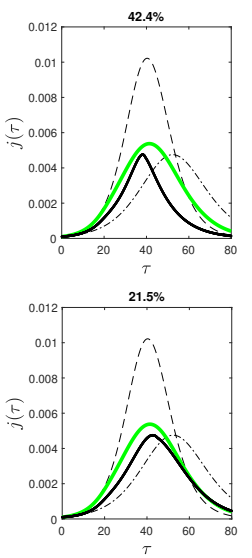

Figure 4. Results for all four versions I-IV. Parameters mentioned in figure.

(I)
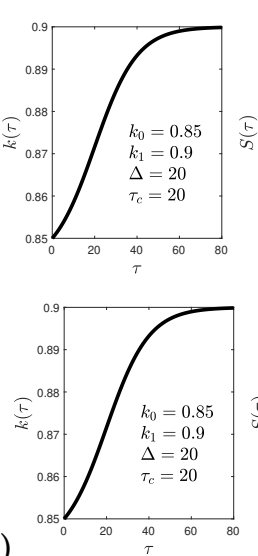

(II)

(III)
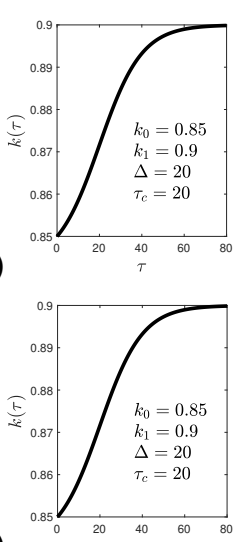
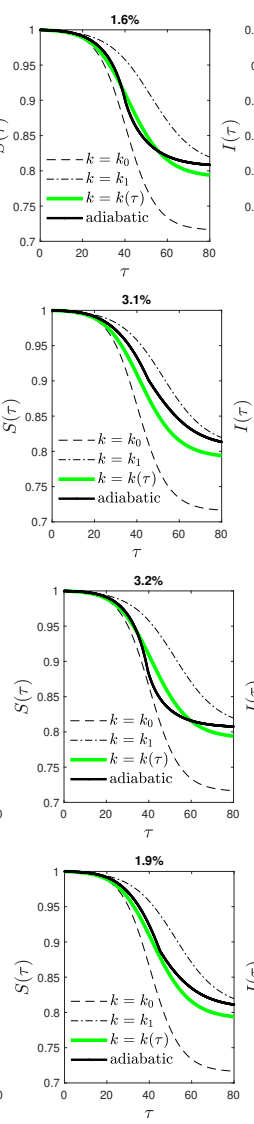

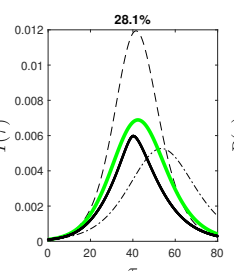

$25 \%$
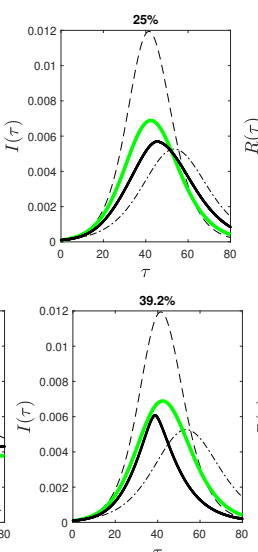

$197 \%$

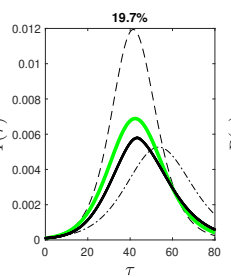

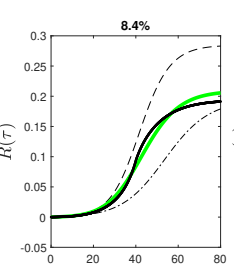

$15.2 \%$

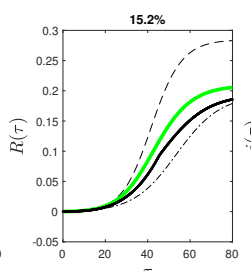

$16.2 \%$

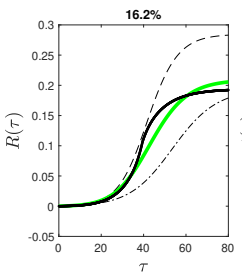

$9.4 \%$

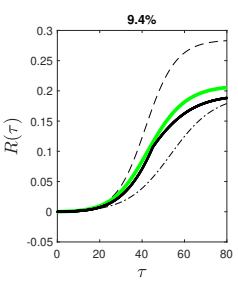

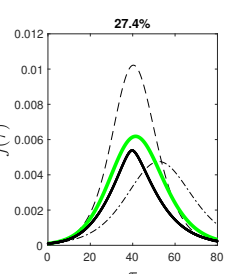

$24.4 \%$

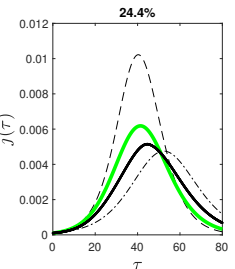

$38.5 \%$

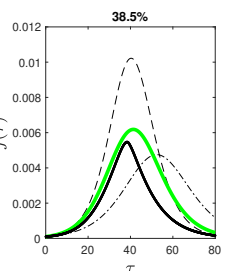

$\stackrel{\tau}{19.5 \%}$

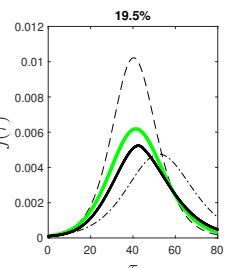

Figure 5. Results for all four versions I-IV. Parameters mentioned in figure. 
(I)

(II)

(III)

(IV)
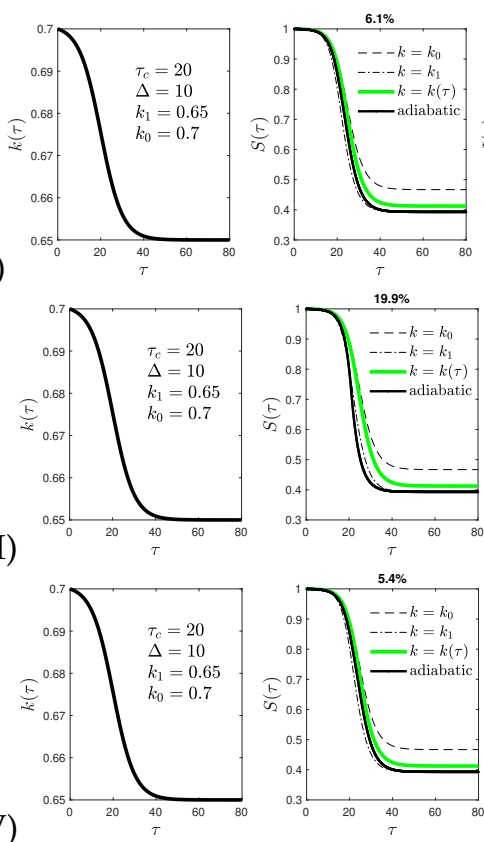
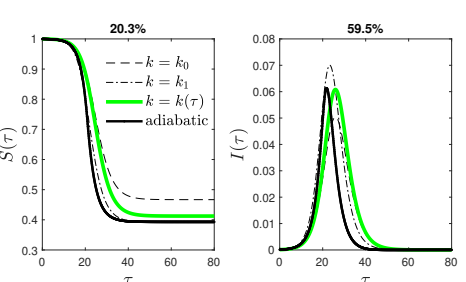

$15.1 \%$

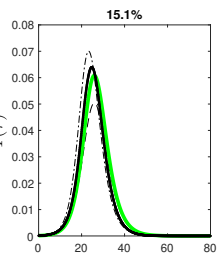

$57.3 \%$
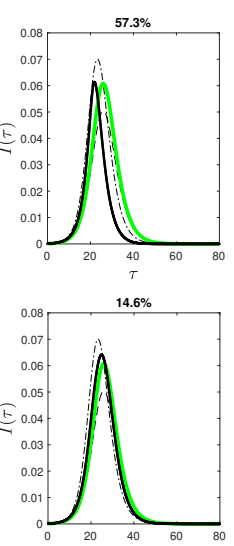
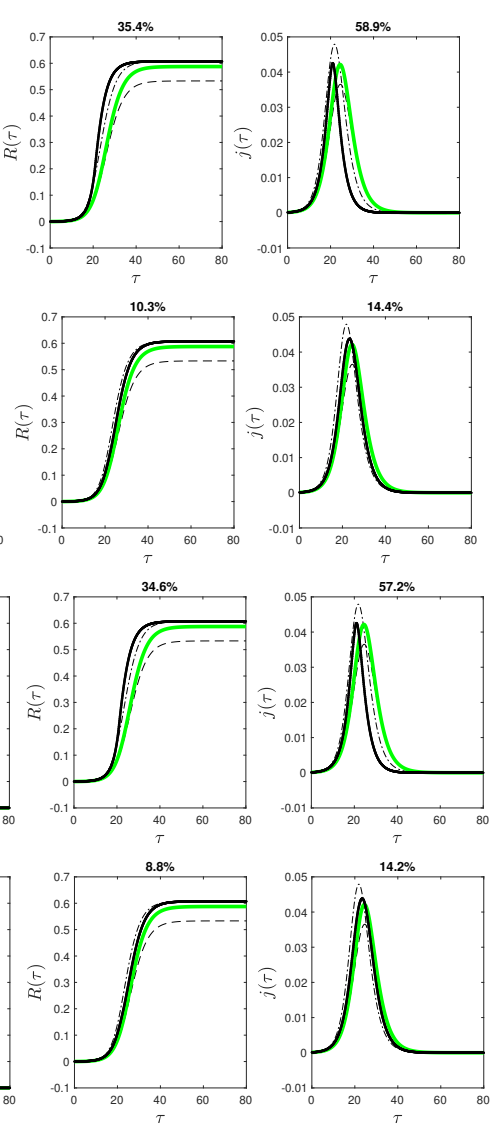

Figure 6. Results for all four versions I-IV. Parameters mentioned in figure.

(I)
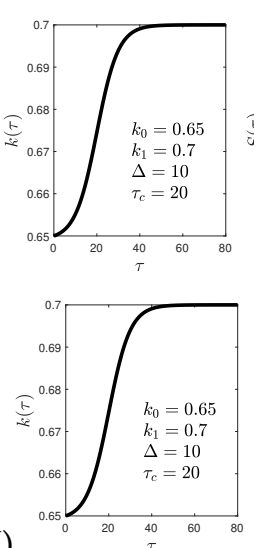

(II)

(III)
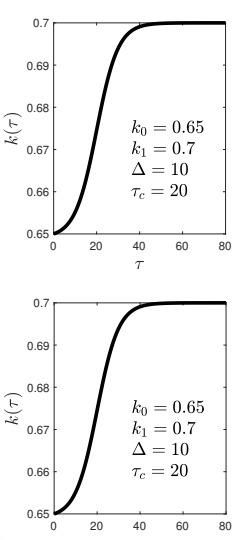
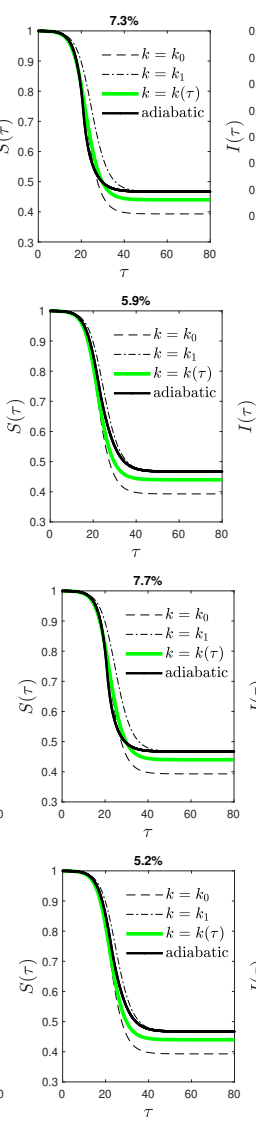
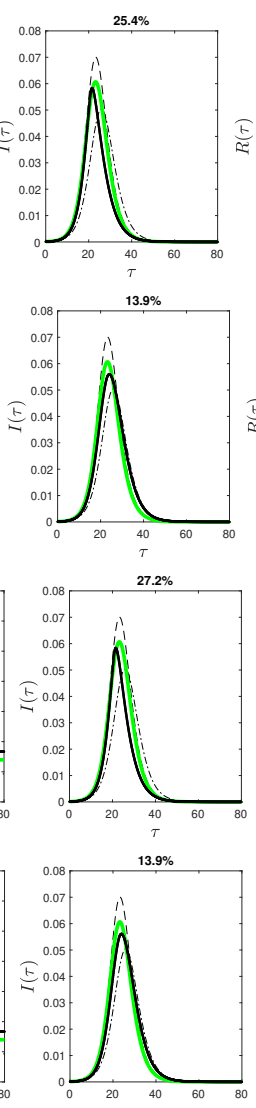
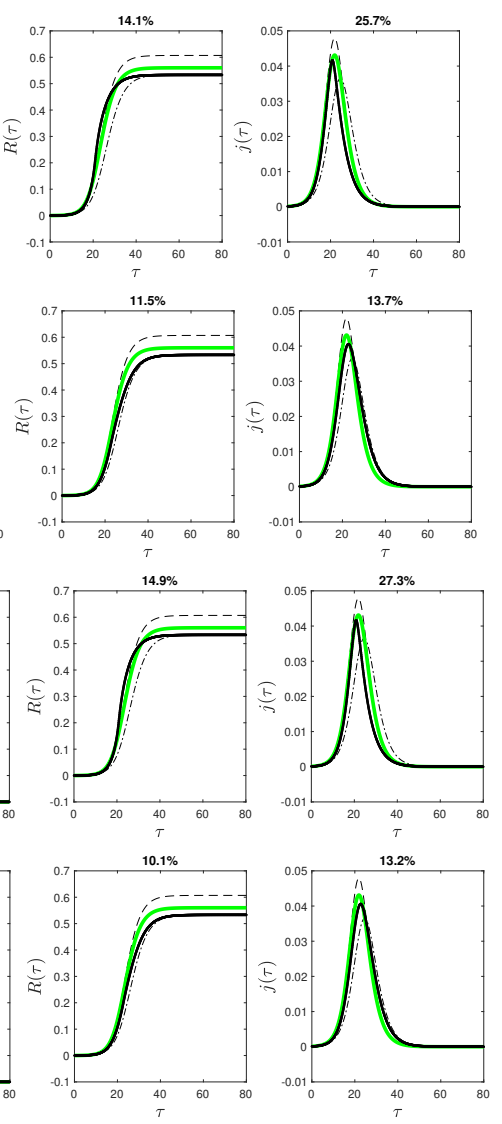

Figure 7. Results for all four versions I-IV. Parameters mentioned in figure. 
(I)
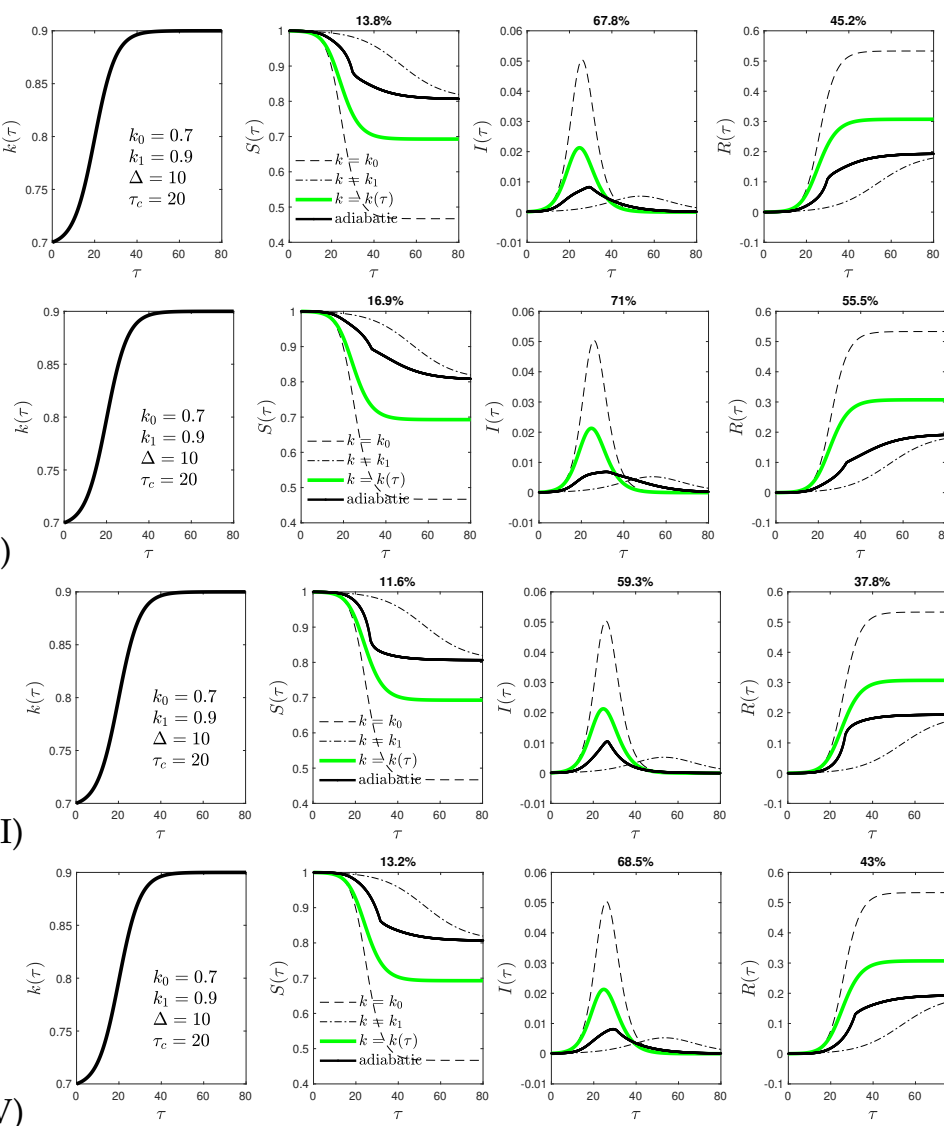

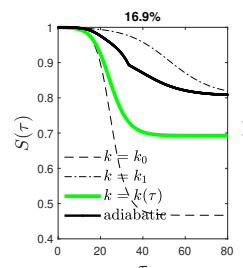

$\tau$
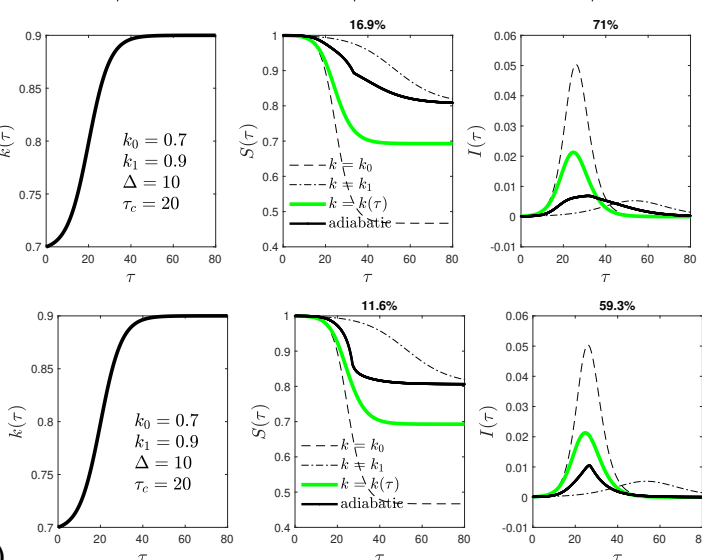

$59.3 \%$

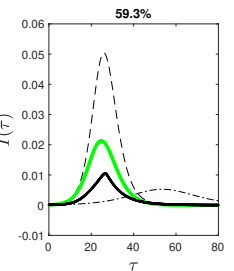

$68.5 \%$
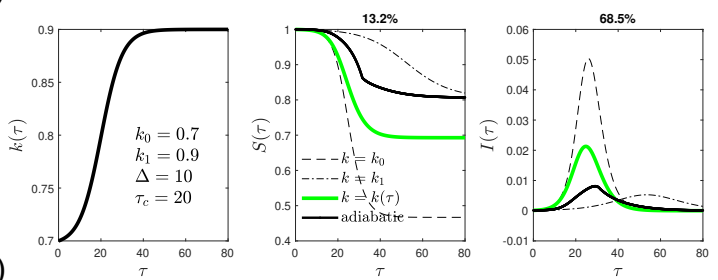

$55.5 \%$

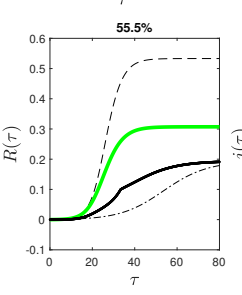

$37.8 \%$

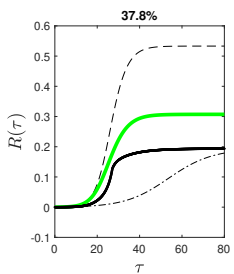

$43 \%$

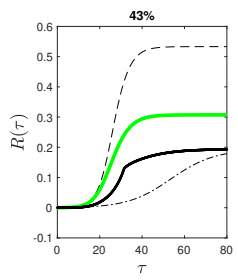

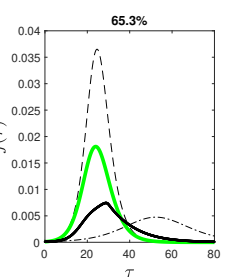

$68.1 \%$

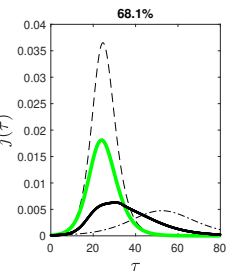

$58.3 \%$

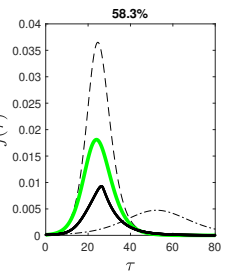

$66.5 \%$

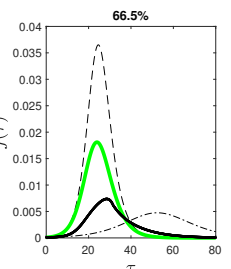

Figure 8. Results for all four versions I-IV. Parameters mentioned in figure.

\section{Relation between the reduced and real time dependence of the infection and recovery rate}

In order to calculate the real time dependence from the reduced time dependence of the SIR-quantities of interest we have to infer the real time dependencies of the infection and recovery rates from the adopted parameterized reduced time dependence (6) of their ratio. We have to consider two different cases: in the first case we adopt a real time dependence of the infection rate $a(t)$, whereas in the second case the real time dependence of the recovery rate $\mu(t)$ is pre-specified. If in the first case a constant infection rate for all real times is adopted, the entire real time variation of the ratio $k(t)$ stems from the recovery rate. Likewise, if in the second case a constant recovery rate at all real times is pre-specified, the real time variation of the rate $k(t)$ results from the infection rate. We consider each case in turn.

\subsection{Case 1: pre-specified infection rate}

In this case we start from constant infection rate $a(t)=a_{0}$ at all times. Then Eq. (3) yields for the reduced time $\tau=a_{0}\left(t-t_{0}\right)$. Consequently, Eqs. (5)-(6) provide for the real time dependence of the recovery rate, i.e.,

$$
\mu(t)=a_{0} k\left(a_{0}\left(t-t_{0}\right)\right)=a_{0}\left\{k_{1}+\frac{k_{1}-k_{0}}{1+\tanh \left(\frac{\tau_{c}}{\Delta}\right)}\left[\tanh \left(\frac{a_{0}\left(t-t_{0}\right)-\tau_{c}}{\Delta}\right)-1\right]\right\} .
$$

For values $k_{1}>k_{0}$ Eq. (18) indeed represents an increase of the recovery rate which can be either slow or rapid depending on the chosen parameters $\Delta$ and $\tau_{c}$. 


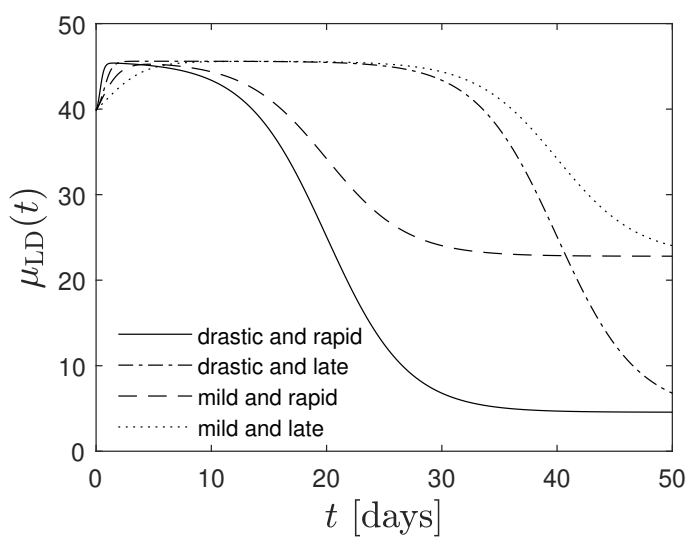

Figure 9. The case of pre-specified lockdown infection rate (19). Shown are the recovery rate $\mu_{\mathrm{LD}}(t)$ versus time $t$ from Eq. (21) using $k_{0}=0.7, k_{1}=0.8, a_{0}=57$ days $^{-1}, t_{b}=7$ days, $\tau_{c}=t_{a}, \Delta=10 q t_{a}$ for the four cases treated earlier: (a) drastic $(q=0.1)$ and rapid $\left(t_{a}=20\right),(b)$ drastic $(q=0.1)$ and late $\left(t_{a}=40\right),(\mathrm{c})$ mild $(q=0.5)$ and rapid $\left(t_{a}=20\right),(\mathrm{d})$ mild $(q=0.5)$ and late $\left(t_{a}=40\right)$.

As a second example of a pre-specified infection rate we consider the earlier considered [33] lockdown infection rate

$$
a_{\mathrm{LD}}(t)=\frac{a_{0}}{2}\left[1+q-(1-q) \tanh \left(\frac{t-t_{0}-t_{a}}{t_{b}}\right)\right] \simeq \begin{cases}a_{0} & \text { for } t_{0} \leq t \ll t_{a} \\ q a_{0} & \text { for } t \gg t_{a}\end{cases}
$$

employing a quarantine factor $q \in[0,1]$, which implies

$$
\begin{aligned}
\tau_{\mathrm{LD}}(t) & =\frac{a_{0}}{2}\left\{(1+q)\left(t-t_{0}\right)-(1-q) t_{b} \ln \left[\frac{\cosh \left(\frac{t-t_{0}-t_{a}}{t_{b}}\right)}{\cosh \left(\frac{t_{a}}{t_{b}}\right)}\right]\right\} \\
& \simeq \begin{cases}a_{0}\left(t-t_{0}\right) & \text { for } t_{0} \leq t \ll t_{a}, \\
q a_{0} t & \text { for } t \gg t_{a} .\end{cases}
\end{aligned}
$$

The time-dependent infection rate (19) has been studied before [33] to describe the effect of lockdown interventions on the temporal evolution of pandemic waves. The function (19) is characterized by four parameters: (i) the initial constant infection rate $a_{0}$ at early times $t \ll t_{a}$, (ii) the final constant infection rate $a_{1}=q a_{0}$ at late times $t \gg t_{a}$ described by the quarantine factor $q=a_{1} / a_{0} \leq 1$, (iii) the time $t_{a}$ of maximum change, and (iv) the time $t_{b} \ll t_{a}$ regularizing the sharpness of the transition. The latter is known to be about $t_{b} \simeq 7$ days reflecting the typical one week incubation delay.

Upon insertion of $a_{\mathrm{LD}}(t)$ and $\tau_{\mathrm{LD}}(t)$ Eqs. (5)-(6) then yield for the real time dependence of the recovery rate

$$
\mu_{\mathrm{LD}}(t)=a_{\mathrm{LD}}(t) k\left(\tau_{\mathrm{LD}}(t)\right) .
$$

In Fig. 9 we show the resulting real time dependence of the recovery rate for the adopted lockdown infection rate.

\subsection{Case 2: pre-specified recovery rate}

The second case of a pre-specified recovery rate is more complex. Starting again from a constant recovery rate $\mu(t)=\mu_{0}$, Eqs. (3), (5)-(6) then yield for the real time dependence of the infection rate the implicit integral equation

$$
a(t)=\frac{\mu_{0}}{k_{1}+\frac{k_{1}-k_{0}}{1+\tanh \left(\frac{\tau_{c}}{\Delta}\right)}\left[\tanh \left(\frac{\int_{t_{0}}^{t} d y a(y)-\tau_{c}}{\Delta}\right)-1\right]}
$$


Introducing

$$
Y(t)=\frac{\int_{t_{0}}^{t} d y a(y)-\tau_{c}}{\Delta}=\frac{\tau-\tau_{c}}{\Delta},
$$

so that $d Y / d t=a(t) / \Delta$, and the abbreviation

$$
K=\frac{k_{1}-k_{0}}{1+\tanh \left(\frac{\tau_{c}}{\Delta}\right)}
$$

Eq. (22) reads

$$
\left(k_{1}-K+K \tanh Y\right) \frac{d Y}{d t}=\frac{\mu_{0}}{\Delta} .
$$

Integrating Eq. (25) then readily yields

$$
\left(k_{1}-k\right) Y(t)+K \ln [\cosh Y(t)]=\frac{\mu_{0}}{\Delta} t+c_{1} .
$$

With the initial value $Y\left(t_{0}\right)=-\tau_{c} / \Delta$ we obtain for the integration constant

$$
c_{1}=-\frac{\mu_{0}}{\Delta} t_{0}-\left(k_{1}-K\right) \frac{\tau_{c}}{\Delta}+K \ln \left[\cosh \left(\frac{\tau_{c}}{\Delta}\right)\right] .
$$

Consequently, Eq. (26) becomes

$$
\left(k_{1}-K\right)\left(Y+\frac{\tau_{c}}{\Delta}\right)+K \ln \left(\frac{\cosh Y}{\cosh \frac{\tau_{c}}{\Delta}}\right)=\frac{\mu_{0}\left(t-t_{0}\right)}{\Delta},
$$

or after inserting Eq. (23),

$$
\left(t-t_{0}\right) \mu_{0}=\left(k_{1}-K\right) \tau+K \Delta \ln \left(\cosh \frac{\tau}{\Delta}-\tanh \frac{\tau_{c}}{\Delta} \sinh \frac{\tau}{\Delta}\right) .
$$

Equation (29) is the resulting relation $t(\tau)$ between the real and reduced times in the case of a constant recovery rate $\mu_{0}$. Using Eq. (29), one can verify that $\tau=\int_{0}^{\tau}\left[\mu_{0} / k(\tau)\right](d t / d \tau) d \tau$ holds.

We consider the limits of Eq. (29) at small and large values of the reduced time $\tau \leq \Delta$ and $\tau \geq \Delta$, respectively. According to Eq. (29) the reduced time $\tau=\Delta$ corresponds to the real time

$$
t_{D}=t_{0}+\frac{\Delta}{\mu_{0}}\left[k_{1}+K \ln \left(\cosh 1-\tanh \frac{\tau_{c}}{\Delta} \sinh 1\right)-K\right],
$$

with $K$ defined in Eq. (24).

\subsubsection{Small real times $t \leq t_{D}$}

For small real times $t \leq t_{D}$ corresponding to reduced times $\tau \leq \Delta$ we use the asymptotics $\sinh (\tau / \Delta) \simeq \tau / \Delta$ and $\cosh (\tau / \Delta) \simeq 1+\left(\tau^{2} / 2 \Delta^{2}\right)$ to approximate to second order

$$
\begin{aligned}
\ln \left(\cosh \frac{\tau}{\Delta}-\tanh \frac{\tau_{c}}{\Delta} \sinh \frac{\tau}{\Delta}\right) & \simeq \ln \left(1-\frac{\tau}{\Delta} \tanh \frac{\tau_{c}}{\Delta}+\frac{\tau^{2}}{2 \Delta^{2}}\right) \\
& \simeq-\frac{\tau}{\Delta} \tanh \frac{\tau_{c}}{\Delta}+\frac{1-\tanh ^{2} \frac{\tau_{c}}{\Delta}}{2} \frac{\tau^{2}}{\Delta^{2}} \\
& =-\frac{\tau}{\Delta} \tanh \frac{\tau_{c}}{\Delta}+\frac{\tau^{2}}{2 \Delta^{2} \cosh ^{2}\left(\frac{\tau_{c}}{\Delta}\right)}
\end{aligned}
$$

In this limit Eq. (29) then yields with the help of abbreviation (24) the quadratic equation

$$
\tau^{2}+\frac{2 k_{0} \Delta \cosh ^{2}\left(\frac{\tau_{c}}{\Delta}\right)}{K} \tau \simeq \frac{2 \mu_{0}\left(t-t_{0}\right) \cosh ^{2}\left(\frac{\tau_{c}}{\Delta}\right)}{K} .
$$


We note that $K>0$ is positive for values $k_{1}>k_{0}$ and negative $K<0$ in the opposite case $k_{1}<k_{0}$. In the more relevant case of positive $K>0 \mathrm{Eq}$. (32) is solved by

$$
\tau\left(t \leq t_{D}\right) \simeq \frac{k_{0} \Delta \cosh ^{2} \frac{\tau_{c}}{\Delta}}{K}\left[\sqrt{1+\frac{2 K \mu_{0}\left(t-t_{0}\right)}{k_{0}^{2} \Delta \cosh ^{2} \frac{\tau_{c}}{\Delta}}}-1\right]
$$

For real times

$$
t-t_{0} \leq \frac{k_{0}^{2} \Delta \cosh ^{2} \frac{\tau_{c}}{\Delta}}{2 K \mu_{0}}
$$

the small time solution (33) approaches the linear relationship

$$
\tau \simeq \frac{\left(t-t_{0}\right) \mu_{0}}{k_{0}}
$$

Differentiating Eq. (33) with respect to $t$ then yields for the real time dependence of the infection rate at small times

$$
a\left(t \leq t_{D}\right) \simeq \frac{\mu_{0}}{k_{0} \sqrt{1+\frac{2 K \mu_{0}\left(t-t_{0}\right)}{k_{0}^{2} \Delta \cosh ^{2} \frac{\tau_{c}}{\Delta}}}}
$$

which decreases for positive $K$ from its initial value $a_{0}=\mu_{0} / k_{0}$.

5.2.2. Large real times $t \geq t_{D}$

Likewise for large real times $t \geq t_{D}$ corresponding to $\tau \geq \Delta$ we insert

$$
\begin{aligned}
\cosh \frac{\tau}{\Delta} & =\frac{e^{\frac{\tau}{\Delta}}}{2}\left(1+e^{-\frac{2 \tau}{\Delta}}\right) \\
\sinh \frac{\tau}{\Delta} & =\frac{e^{\frac{\tau}{\Delta}}}{2}\left(1-e^{-\frac{2 \tau}{\Delta}}\right)
\end{aligned}
$$

so that in this limit

$$
\begin{aligned}
\ln \left[\cosh \frac{\tau}{\Delta}-\tanh \frac{\tau_{c}}{\Delta} \sinh \frac{\tau}{\Delta}\right] & =\frac{\tau}{\Delta}-\ln (2)+\ln \left(1-\tanh \frac{\tau_{c}}{\Delta}\right)+\ln \left[1+\frac{1+\tanh \frac{\tau_{c}}{\Delta}}{1-\tanh \frac{\tau_{c}}{\Delta}} e^{-\frac{2 \tau}{\Delta}}\right] \\
& \simeq \frac{\tau}{\Delta}-\ln (2)+\ln \left(1-\tanh \frac{\tau_{c}}{\Delta}\right)+\frac{1+\tanh \frac{\tau_{c}}{\Delta}}{1-\tanh \frac{\tau_{c}}{\Delta}} e^{-\frac{2 \tau}{\Delta}}, \quad \text { (38) }
\end{aligned}
$$

and consequently, at late times, Eq. (29) can be approximated as

$$
\tau+\frac{\Delta\left(k_{1}-k_{0}\right)}{k_{1}\left(1-\tanh \frac{\tau_{c}}{\Delta}\right)} e^{-2 \frac{\tau}{\Delta}} \simeq \frac{\mu_{0}\left(t-t_{0}\right)+K \Delta\left[\ln 2-\ln \left(1-\tanh \frac{\tau_{c}}{\Delta}\right)\right]}{k_{1}} .
$$

Because at late time $\tau \geq \Delta$ the second term on the left-hand side of Eq. (39) is much smaller than the first term one obtains as approximate solution of Eq. (30)

$$
\begin{aligned}
\tau\left(t \geq t_{D}\right) \simeq & \frac{\mu_{0}\left(t-t_{0}\right)+K \Delta\left[\ln 2-\ln \left(1-\tanh \frac{\tau_{c}}{\Delta}\right)\right]}{k_{1}} \\
& -\frac{\Delta\left(k_{1}-k_{0}\right)\left(1-\tanh \frac{\tau_{c}}{\Delta}\right)^{\frac{2 K}{k_{1}}-1}}{k_{1} 2^{\frac{2 K}{k_{1}}}} e^{-\frac{2 \mu_{0}\left(t-t_{0}\right)}{\Delta k_{1}}},
\end{aligned}
$$

and the real time derivative of this equation then provides for the infection rate at late times

$$
a\left(t \geq t_{D}\right) \simeq \frac{\mu_{0}}{k_{1}}\left[1+\frac{k_{1}-k_{0}}{k_{1}}\left(\frac{1-\tanh \frac{\tau_{c}}{\Delta}}{2}\right)^{\frac{2 K}{k_{1}}-1} e^{-\frac{2 \mu_{0}\left(t-t_{0}\right)}{\Delta k_{1}}}\right],
$$




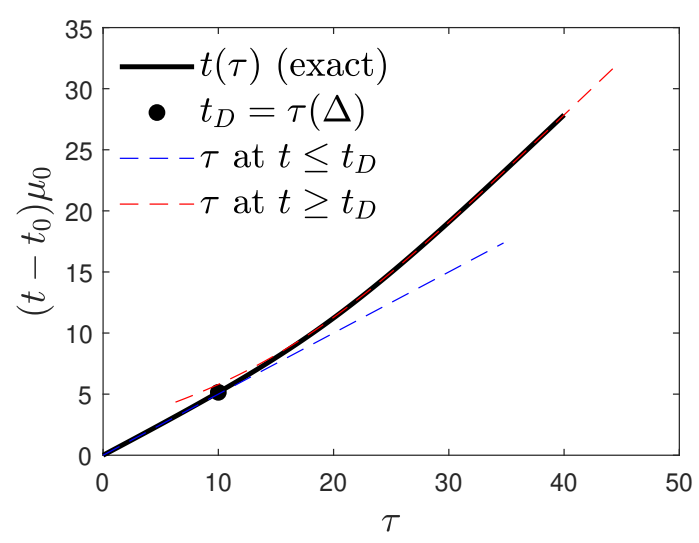

Figure 10. Real time $t(\tau)$ versus $\tau$ (solid black) for the case of $k_{0}=0.5, k_{1}=0.9, \tau_{c}=20, \Delta=10$ at fixed constant infection rate $\mu_{0}$. Highlighted is the transition time $t_{D}$ given by Eq. (30), and the asymptotic expressions (35) and (40) for small and large times, respectively.

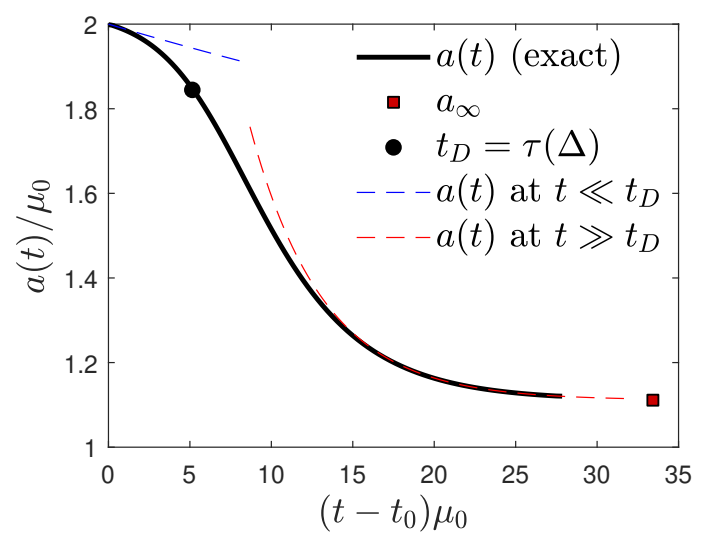

Figure 11. Infection rate $a(t)$ versus real time $t$ (solid black) for the parameters used in Fig. 10 . Highlighted is the transition time $t_{D}$ given by Eq. (30), the asymptotic expressions (36) and (41) for small and large times, respectively, as well as $a_{\infty}=\mu_{0} / k_{1}$.

which decreases to its final value $a_{\infty}=\mu_{0} / k_{1}$ in the limit $t \rightarrow \infty$ for positive values of $K>0$ corresponding to $k_{1}>k_{0}$. In Figs. (10) and (11) we compare the exact real time dependencies $t(\tau)$ as a function of the reduced time $\tau$ and the real time dependence of the infection rate $a(t)$, respectively, with their analytical approximations at small and large real times for the case of a constant recovery rate. The agreement between the exact results and the approximations is remarkably good.

\subsubsection{Variable recovery rate $\mu(t)$}

We end this subsection by noting that for a general variable but pre-specified real time dependence of the recovery rate $\mu(t)$ Eq. (29) reads instead

$$
\int_{t_{0}}^{t} d t^{\prime} \mu\left(t^{\prime}\right)=\left(k_{1}-K\right) \tau+K \Delta \ln \left(\cosh \frac{\tau}{\Delta}-\tanh \frac{\tau_{c}}{\Delta} \sinh \frac{\tau}{\Delta}\right) .
$$

Only if the functional real time dependence $\mu(t)$ is given, this equation can be analyzed further.

\subsection{Real time dependence of the rate of new infections for a constant recovery rate}

Because we regard the case of a pre-specified constant recovery rate and a real time dependent infection rate as important we calculate in this case the resulting real time dependence of the rate of new infections 


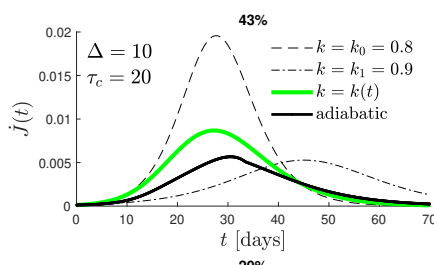

$20 \%$

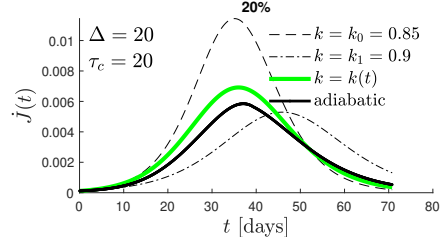

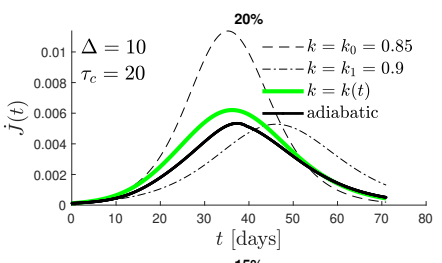

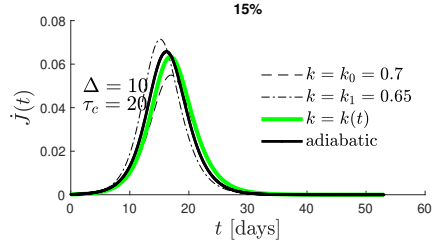

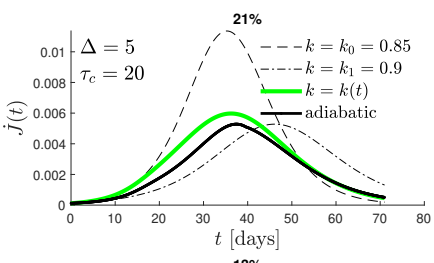

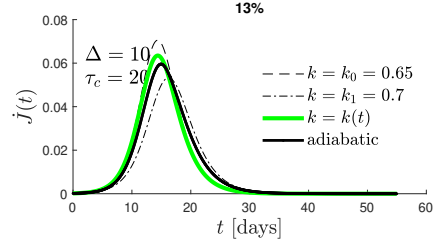

Figure 12. Daily new rate of infected persons $\dot{J}(t)$ versus real time $t$ in the case of a constant recovery rate according to Eq. (43) for parameters specified in the panels. Each panel belongs to one of the former figures where we showed $S, I, R$, and $j$ versus reduced time $\tau$. The adiabatic version IV (solid green) is used for comparison with the exact numerical solution (solid black), and the reference cases of constant $k(t)=k_{0}$ (dashed) and $k(t)=k_{1}$ (dash-dotted) are shown as well.

$$
\dot{J}(t)=a(t) j(\tau(t))
$$

using the results for $a(t)$ and $\tau(t)$ from the last subsection. In Fig. 12 we show the adiabatic approximation from version IV in comparison with the exact numerical results and also the limiting KSSIR-variations adopting the initial ratio $k_{0}$ and final ratio $k_{1}$ at all times.

\section{Summary and conclusions}

The temporal evolution of pandemics described by the SIR-compartment model is sensitively determined by the time dependence of the infection $(a(t))$ and recovery $(\mu(t)$ rates. These two rates regulate the transitions from the susceptible to the infected compartment and from the infected to the recovered compartment, respectively. Starting from the pioneering work [1,2] many numerical solutions of the SIR equations have used stationary values of the two rates, an assumption also made in the analytical solution by Harko et al. [7]. An important improvement regarding analytical SIR-solutions has been provided by the KSSIR-model [8,9] holding for arbitrary but given time dependencies of the infection rate $a(t)$ for the case of a stationary ratio $k=\mu(t) / a(t)$, implying that the recovery rate has exactly the same time dependence as the infection rate. This generalization to a time-dependent infection rate is important as such time-dependencies are caused by non-pharmaceutical interventions (NPIs) taken during pandemic outbreaks.

In this work, apparently for the first time, we derive approximated SIR-solutions for different time dependencies of the infection $(a(t))$ and recovery $(\mu(t))$ rates so that their ratio no longer is constant and becomes also time-dependent. Our analysis is based on the adiabatic approximation assuming time-dependent ratios $k(t)$, slowly varying in comparison to the typical time characteristics of the pandemic wave. For such slow variations we use the available analytical approximations from the KSSIR-model and insert a-posteriori the adopted time-dependent ratio of the two rates.

Instead of investigating endless different combinations of the time dependencies of the two rates $a(t)$ and $\mu(t)$ we adopt a suitably parameterized reduced time dependence of the ratio $k(\tau)$. Together with the definition of the reduced time this parameterized ratio $k(\tau)$ allows us to represent a great variety of different time dependencies of the infection and recovery rates. This includes the important case of a stationary recovery rate but a time-dependent infection rate which is investigated in detail.

In each considered case the obtained solutions from the adiabatic approximation in its four different studied variants are compared to the exact numerical solutions of the SIRequations. In the worst case the maximum deviation is $68.1 \%$ but in most shown examples much less. This remarkable good agreement provides strong confidence in the accuracy 
of the proposed adiabatic approximation. The adiabatic approximation works best for small differences $\left|k_{1}-k_{0}\right|$ between the final $\left(k_{1}\right)$ and initial $\left(k_{0}\right)$ values of the function $k(\tau)$; the maximum deviation for the rate of of new infections scales roughly as $\simeq 400\left|k_{1}-k_{0}\right|$ percent.

Author Contributions: Conceptualization, R.S.; methodology, R.S. and M.K.; software, M.K.; investigation, R.S. and M.K.; writing-original draft preparation, R.S.; writing—review and editing, M.K.; All authors have read and agreed to the published version of the manuscript.

Funding: This research received no external funding.

Data Availability Statement: All data required to reproduce the results are part of the present manuscript.

Conflicts of Interest: The authors declare no conflict of interest.

\section{References}

1. Kermack, W.O.; McKendrick, A.G. A contribution to the mathematical theory of epidemics. Proc. R. Soc. A 1927, 115, 700.

2. Kendall, D.G. Deterministic amd stochastic epidemics in closed populatuions; University of California Press: Berkeley, CA, USA, 1956; Vol. 4, p. 149.

3. Hethcode, H.W. The mathematics of infectious deseases. SIAM Rev. 2000, 42, 599.

4. Keeling, M.J.; Rohani, F. Modeling Infectious Diseases in Humans and Animals; Princeton University Press: Princeton, NJ, USA, 2008.

5. Schlickeiser, R.; Kröger, M. Analytical modelling of the temporal evolution of epidemics outbreaks accounting for vaccinations. Physics 2021, 3, 386.

6. Estrada, E. COVID-19 and SARS-CoV-2. Modeling the present, looking at the future. Phys. Rep. 2020, 869, 1.

7. Harko, T.; Lobo, F.S.N.; Mak, M.K. Exact analytical solution of the susceptible-infected-recovered (SIR) epidemic model and of the SIR model with equal death and birth rates. Appl. Math. Comp. 2014, 236, 184.

8. Kröger, M.; Schlickeiser, R. Analytical solution of the SIR-model for the temporal evolution of epidemics. Part A: Timeindependent reproduction factor. J. Phys. A 2020, 53, 505601.

9. Schlickeiser, R.; Kröger, M. Analytical solution of the SIR-model for the temporal evolution of epidemics: Part B. Semi-time case. J. Phys. A 2021, 54, 175601.

10. Kröger, M.; Schlickeiser, R. Verification of the accuracy of the SIR model in forecasting based on the improved SIR model with a constant ratio of recovery to infection rate by comparing with monitored second wave data. R. Soc. Open Sci. 2021, 8, 211379.

11. Schlickeiser, R.; Kröger, M. Forecast of omicron wave time evolution. Covid 2022, 2, 216-229.

12. Lerche, I. Epidemic evolution: Multiple analytical solutions for the SIR model. preprints.org 2020, p. 2020080479. doi:10.20944/preprints202008.0479.v1.

13. Schoner, G.; Haken, H. A systematic elimination procedure for Ito stochastic differential-equations and the adiabatic approximation. Z. Physik B 1987, 68, 89-103.

14. Yukalov, V.I. Adiabatic theorems for linear and nonlinear Hamiltonians. Phys. Rev. A 2009, 79.

15. Wentzel, G. Eine Verallgemeinerung der Quantenbedingungen für die Zwecke der Wellenmechanik. Z. Physik 1926, 38, 518-529.

16. Kramers, H.A. Wellenmechanik und halbzahlige Quantisierung. Z. Physik 1926, 39, 828-840.

17. Brillouin, L. La mécanique ondulatoire de Schrödinger: une méthode générale de resolution par approximations successives. Compt. Rendus l'Acad. Sci. 1926, 183, 24-26.

18. Jeffreys, H. On certain approximate solutions of linear differential equations of the second order. Proc. London Math. Soc. 1923, 23,428 .

19. Born, M.; Oppenheimer, R. Zur Quantentheorie der Molekeln. Ann. Phys. 1927, 389, 457.

20. Fricke, L.M.; Glockner, S.; Dreier, M.; Lange, B. Impact of non-pharmaceutical interventions targeted at COVID-19 pandemic on influenza burden - a systematic review. J. Infection 2021, 82, 1.

21. Kasting, M.L.; Head, K.J.; Hartsock, J.A.; Sturm, L.; Zimet, G.D. Public perceptions of the effectiveness of recommended non-pharmaceutical intervention behaviors to mitigate the spread of SARS-CoV-2. PLoS One 2020, 15.

22. Ullah, S.; Khan, M.A. Modeling the impact of non-pharmaceutical interventions on the dynamics of novel coronavirus with optimal control analysis with a case study. Chaos Solitons E Fractals 2020, 139, 110075.

23. Flaxman, S.; Mishra, S.; Gandy, A.; Unwin, H.J.T.; Mellan, T.A.; Coupland, H.; Whittaker, C.; Zhu, H.; Berah, T.; Eaton, J.W.; et al. Estimating the effects of non-pharmaceutical interventions on COVID-19 in Europe. Nature 2020, 584, 257+.

24. Hsiang, S.; Allen, D.; Annan-Phan, S.; Bell, K.; Bolliger, I.; Chong, T.; Druckenmiller, H.; Huang, L.Y.; Hultgren, A.; Krasovich, E.; et al. The effect of large-scale anti-contagion policies on the COVID-19 pandemic. Nature 2020, 584, $262+$.

25. Nicola, M.; O’Neill, N.; Sohrabi, C.; Khan, M.; Agha, M.; Agha, R. Evidence based management guideline for the COVID-19 pandemic-Review article. Int. J. Surgery 2020, 77, 206-216. 
26. Eikenberry, S.E.; Mancuso, M.; Iboi, E.; Phan, T.; Eikenberry, K.; Kuang, Y.; Kostelich, E.; Gumel, A.B. To mask or not to mask: Modeling the potential for face mask use by the general public to curtail the COVID-19 pandemic. Infect. Disease Model. 2020, 5, 293-308.

27. Merler, S.; Ajelli, M.; Fumanelli, L.; Gomes, M.F.C.; Pastore y Piontti, A.; Rossi, L.; Chao, D.L.; Longini, Jr., I.M.; Halloran, M.E.; Vespignani, A. Spatiotemporal spread of the 2014 outbreak of Ebola virus disease in Liberia and the effectiveness of non-pharmaceutical interventions: a computational modelling analysis. Lancet Infect. Diseases 2015, 15, $204-211$.

28. Ferguson, N.M.; Cummings, D.A.T.; Fraser, C.; Cajka, J.C.; Cooley, P.C.; Burke, D.S. Strategies for mitigating an influenza pandemic. Nature 2006, 442, 448-452.

29. Kröger, M.; Schlickeiser, R. Gaussian doubling times and reproduction factors of the COVID-19 pandemic disease. Frontiers Phys. 2020, 8, 276.

30. Haas, F.; Kröger, M.; Schlickeiser, R. Multi-hamiltonian structure of the epidemics model accounting for vaccinations and a suitable test for the accuracy of its numerical solvers. J. Phys. A 2022. submitted Nov 2021, under review.

31. Shampine, L.F.; Hosea, M.E. Analysis and implementation of TR-BDF2. Appl. Numer. Math. 1996, 20, 21-37.

32. Shampine, L.F.; Reichelt, M.W.; Kierzenka, J.A. Solving index-1 DAEs in matlab and simulink. SIAM Rev. 1999, 41, 538-552.

33. Schlickeiser, R.; Kröger, M. Epidemics forecast from SIR-modeling, verification and calculated effects of lockdown and lifting of interventions. Front. Phys. 2021, 8, 593421. 